\title{
Analysis on Relationship Between Painting and Animation
}

\author{
Li yongjun, Li Wei \\ Weifang University of Science \& Technology, Shandong, Shouguang262700
}

\begin{abstract}
In the 21 st century, the development of revolution in the new era emphasizes the development of universal art, and the cartoon develops rapidly in China, which realizes effective inheritance and development of painting and animation. How to ensure that painting and cartoon has better visual art and appreciation value is one the focuses of the animation industry in China. While researching and analyzing painting and animation, the paper firstly analyzes the principle and main manifestation of animation, and then compares painting and animation. Lastly, the paper expounds the relationship between painting and animation.
\end{abstract}

Key words: painting, animation, compare, relationship

\section{INTRODUCTION}

As the premise of animation, painting not only is the important basic link of animation, but also is an important guarantee of animation. While animation develops rapidly, it pays more attention to pursuing the artistic beauty and value of pictures, and the different animation is constructed based on watercolor, which not only realizes transcendence of visual design, but also promotes the development of animation industry and improves the core status of national economic development in China. So analyzing the relationship between animation and painting has great economic value and realistic significance.

\section{Basic Principle and Manifestation of Animation}

Animation is an artistic manifestation of film. It is different from live-action film, and it has the same artistic manifestation like film, and unique emergence of artistic form, it has the characteristics of humor, which makes it become an universal culture and is popular with the people in the country.

(1) Basic principle of animation

The reason why animation industry develops rapidly is that the design technique makes great progress. And the present manufacturing technology and style is different. While computer develops, animation technique tends to develop towards digital life. It breaks through traditional manual painting, and implements the technical innovation based on traditional animation. It combines traditional animation, which shows a colorful effect.

(2) Manifestation of animation

The existence of animation is not only a form. There present animation is generally divided into two-dimensional animation, three-dimensional animation and claymation. For two-dimensional animation, it emphasizes the effect of manual painting and great decoration beauty. But the manual painting generally needs the help of art designer to paint generous pictures, and the completion of workload needs the help of computers. Three-dimensional painting creates the animation with the help of computers and software of three-dimensional animation. Three-dimensional animation has great pursuit for space sense and volume sense. It establishes three-dimensional model of objects and background with the help of three-dimensional animation manufacturing technique. And the motivation of the objects is shot. Under the rendering of light, a lively picture is shown. Claymation is a hand-drawn animation manifestation. It is a form manufacturing animation images with the help of clay and rubber. 


\section{Comparison of Painting and Animation}

The early practical scene change of animation generally has great painting. With the development of computer technique and digital process, in the process of inheriting art element and form of painting, animation is the continuity of painting time, and it shapes the new artistic form and aesthetic image. For the essence, animation and painting has general characteristics and difference.

(1) Similarity of painting and animation

Painting and animation has the same function, which means that the audience know more about the life styles and behaviors of people with different nations in different times by enjoying the art and animation works. The painting and animation has the same education function, which is a manifestation of thought and morality of the audience. And it plays the role of education and inflection with the help of a content and a theme. As an ornamental artistic work, painting and animation generally has the same aesthetic function. While cultivating the discrimination of the people, it improves aesthetic state and taste of people. The artistic form factors can show the unique charm of visual art, and it makes the people have dedicate feeling by art process of dynamic and static combination. The manifestations which are identical have different expressions. In the process of showing works, animation works generally need the help of watercolor and sketch of painting, which realizes the continuity of painting with the help of animation. For painting, animation has mature art. Animation process shows a process, which is limited by the time. Animation is a dynamic painting to some extent. But painting is only a static animation performance. In the application of cartoons, the sketch and watercolor of painting not only is universal, but also supplements each other, which shows unique charm of art together.

(2) Difference of painting and animation

Generally speaking, animation is a continuity of painting, and painting is a state of static animation. But the substantiality of animation and painting is unique.

Firstly, the modeling of painting and animation has great difference. The character image design of animation generally has certain unique style, and applies background painting. And role shaping is a decoration of style. Secondly, painting and animation is difference for actual composition of pictures. Painting is a manifestation on the incidents, but animation is a reflection on the action. In the actual composition of pictures, painting is a or more composition of pictures, but animation is not single in the actual composition of pictures. In gradual push and pull of scenes, animation has varied composition of picture. For the materiality, the continuity and dependence of painting and animation picture is different. The continuity and dependence of painting needs the help of single picture, which has dependent manifestation style. In actual performance process, animation has many pictures and has continuous dynamic performance. And there is close correlation in the performance of animations.

Above all, animation and painting not only is universal, but also has difference in actual development. So unique art perspective is established.

\section{Relationship Between Painting and Animation}

Painting and animation has close relationship in the process of development. The composition of picture, color and modeling of animation and painting has direct relationship. The relationship between painting and animation can be analyzed from the 
perspective of modeling, composition of picture and colors.

(1) Relationship of modeling between animation and painting

Animation is an art of visual communication, and painting is an unique art of visual communication. Animation has different relationship with single art creation to some extent, and it is an expression of personal emotion in actual performance. It not only has the restriction of plot, but also has the limitation of script theme and role model. The first process of animation is painting, which is an important link transforming imagination into the fact. The model of painting has direct relationship with animation to some extent. The painting of body structure is an exaggerated skill which fully knows the structure of body, fully masters the proportion of body, increases the interest of painting with the help of metabolic design method, and rationally controls various dynamic changes of body. The local painting of body emphasizes the expressions of animation figures, which increases the inflection of art. In the present animation design, the expression of body emotion and external and internal beauty of body realizes rational comparison of figures with the help of eyes.

(2) Relationship of composition of picture between animation and painting

In traditional painting, the first task of picture design is rational composition of picture, which determines the relationship of compositions of pictures by actual composition of picture, constitutes the unified picture, processes positional relationship with the help of basic principle of appreciation of the beauty, and makes reasonable creation of composition of a picture. Selection of composition perspective in painting plays the role of creating the atmosphere of animation works. In actual visual process, it reflects lines and colors with the help of simple scenes. In the process of creating pictures, it emphasizes the change of perspective view. The relationship of distances and the proportion of space is set rationally to reflect three-dimensional space, which not only ensures that composition of pictures is various, but also embodies the perspective relationship of space. For the animation work, Madagascar, in actual scene building process, by determining the end point on eye level of pictures, Keane determines the direction of perspective rays in pictures, and draws the outline of buildings. The actual composition rule and method of animation and painting has correlation. And the selection of perspective is based on the theories of modern painting.

(3) Relationship of colors between animation and painting

The selection of animation color emphasizes rational control of model language, which makes visual stimulation on human senses, enhances the culture and actual interest, and increases the beauty of design space. In the control of colors, the completion of works is realized with the help of some dynamic stories in animations. There are some color rules in painting. With the help of color rules, the ornament of animation art is improved, the effect of pictures is considered, and the color emotion is set off from the perspective of physiological equilibrium and psychological requirements. In actual application, painting emphasizes the creation of atmosphere, and reflects the flexibility and pertinence of live-action film.

\section{Conclusion:}

In conclusion, as the continuity of traditional painting, animation is the inheritance of the essence of painting. As the pioneer of animation, painting is the basic force of animation works. Animation and painting works not only have certain similarity to some extent, but also have difference. In the present development, animation and painting has close relationship, and supports the comprehensive development of animation industry together. 
References:

[1] Li Peng, Inheritance of Chinese traditional painting art in animation modeling design [J], Grand Stage, 2012,08:156-157.

[2] Wang Xiao, Analysis on difference of the current animation and film $[\mathrm{J}]$, Film Literature, 2012,16:55-56.

[3] Dai Dan, From poetry and painting to painting and rhetoric — a transformation in art history research [J], Journal of Nanjing Art College (Art and Design
Edition), 2014,03:22-28+187.

[4] Xing Jie, Chang Hong, Research on transmutation of animation art in digital media era [J], Contemporary Film, 2014,05:167-170.

[5] Luo Songyi, Application of figure painting in animation film in China $[\mathrm{J}], \quad$ Film Literature,2011,19:65-66.

[6] Zhang Xiang, Observation on traditional painting elements in animation model creation in China [J], Popular Literature, 2013,19:116-117. 